\title{
RELIABLE DATA COLLECTOR BASED EFFICIENT DATA COLLECTION FOR IMPROVING ENERGY EFFICIENCY IN WSN
}

\author{
G. Vishnupriya
}

Department of Computer Science and Engineering, Sathyabama Institute of Science and Technology, Chennai, India, vishnupriya12phd@gmail.com

R. Ramachandran

Department of Electronics and Communication Engineering, Dhanalakshmi College of Engineering, Chennai,

Abstract

Wireless Sensor Network (WSN) is efficient for data collection and communication. Generally, sensor nodes observed the data has amount of noises otherwise duplicate presents, hence, the data are collected to take out significant information also diminish the communication expenditure. In this paper, an Reliable Data Collector based Efficient Data Collection for Improving Energy Efficiency in WSN (REDC) for Improving Energy Efficiency (EE) that powerfully minimizes duplicate as well as incorrect data. A Bio-Inspired Method (BIM) is used for selecting reliable data collector in the WSN. The BIM method like Ant Colony Optimization (ACO) technique achieves an efficient path leading to the most reputed node in a WSN. Bivariate polynomials key verification is used for verifies the reliable collector. Active list will be generated in filtering node to accumulate history messages yet communicated through this node. The entire messages will be estimated whether repeated duplicated or not along with the messages in list. Simulation results illustrates this approach increases the data accuracy ratio and EE in the network.

Keywords: Energy Efficiency; Wireless sensor network; Clustering, Bivariate polynomials key verification; Ant Colony Optimization; Active Message List; Filtering Node; Filtering redundancy.

\section{Introduction}

WSN is broadly distributed at the present time on a bulk range of applications. The significant aim of a sensor network is to gather information concerning a set of developments. This development is little because batteries' life is imperfect also hence communication and computing functions should be denigrated. A frequent job in sensor network is to assess the observed information as well as to increase the calculated samples throughout the network. Therefore, time series evaluation approaches are fundamental for this kind of works so as to decrease data communication. Energy utilization can be diminished utilizing data collection method that collected of clustering occurrence, helpful for broad variety of engineering, ecological, farming as well as armed applications that mostly admits applications similar to temperature, smart cities additionally moisture observing, flood recognition, RADAR exposure, etc. In clustering, every cluster has a Cluster Head (CH) that collect information and transmit it to the Base Station (BS) for that size of cluster as well as data communication rate are imperative features [Haghighi et al, 2014].

In WSNs, the energy utilization owing to the observed data communication is extra than dispensation data nearby inside the sensor node. If the affect owing to duplicate as well as noisy information can be diminished, the reserve operation also network function will be considerably raised [Gao et al, 2019]. The data collection is one of the proficiencies to preserve energy through rejecting the duplicate data communication also transmit significant information to the BS [Nakas et al, 2020]. Data collection is the approaches to diminish the transaction weight in that a sensor node naming data collector processes and collects received data before transient it to its neighbour node. Data collection is an important method to attain energy efficiency through minimizing data redundancy as well as optimize the utilization of bandwidth [Bista and Chang, 2010].

Bio-inspired technique that is encouraged through the behavior of nature utilized for executing optimization. It is utilized for enhancing the efficiency as well as accuracy [Raychaudhuri and De, 2020]. In this approach Particle Swarm Optimization (PSO) with Bacterial Foraging Optimization (BFO) techniques which can efficiently accelerate the convergence rate. BFO technique that can enhance the global searching ability. PSO and ACO are applying a significant task in solve the problems of WSN [Shayokh and Shin, 2017]. Data aggregation tree approach (DATA) is used to diminish the energy expenditure for communicating data in the network. Throughout in-between enquiry region, the sensory as well as collection information have been 
retorted via an amount of dissimilar transmitting trees inside every sub query area [Wan et al, 2016]. However, this approach data collection accuracy very less. In addition, selection of data aggregator is not efficiently.

To solve these problems, this article Reliable Data Collector based Efficient Data Collection is proposed. It Improving energy efficiency and it powerfully minimizes duplicate as well as incorrect data. Here, we using BIM method like ACO technique achieves an efficient path leading to the most reputed node. Data collector reliability is verified by Bivariate polynomials key verification method. Active list will be generated in filtering node to accumulate history messages yet communicated through this node. The entire messages will be estimated whether repeated duplicated or not along with the messages in list. It improves the data collector accuracy.

\section{Related Work}

Energy Efficient Data Aggregation Scheme is used for minimizing intra-cluster transactions via granting cluster member to transmit small sized control frames adopted through moderately complete frames from nodes particular through the $\mathrm{CH}$ node [Roy and Chandra, 2019]. A single-hop clustering method, in that sensor nodes are formed into clusters also transmit with a destination via a single hop. This approach established on the autoregressive, affecting usual, as well as tempered least mean square examples to forecast local perceived samples so as to decrease wireless data transaction [Waghmare et al, 2016]. EE structure-free data aggregation and delivery approach that collects the repeated information in the middle nodes. In this approach, a waiting period for data packets by every middle node is computed logically thus which information can be collected resourcefully in the routing path. The supervised information packets are communicated sensibly to the collection point for information collection. The buffer of every node is zoned to preserve dissimilar kinds of streams for fair also capable data deliverance. The communication rates of the sources as well as middle nodes are used to during congestion [Miranda and Ramos, 2016].

Cluster-chain mobile agent routing based Data collection is an intellectual method where the information from dissimilar sources are collected at relay nodes, thus diluting the amount of packets to be transmit to the destination. It creates full utilize of the benefits of both least energy adaptive clustering as well as proficient collecting in the network [Sasirekha and Swamynathan, 2017]. The Chinese Remainder Theorem (CRT) based data collection method utilizing a disseminated approach with no necessitate of coordination between nodes. In addition, a suitable network coding method established on the CRT is joint to source coding to facilitate lossless communication from the sender to the destination [Campobello et al, 2013]. An estimation technique is introduced to assemble a data collection tree whose opposite lifespan is certain to be within a hop from the best one. Adaptable communication energy levels of the sensors establish an extra condition in the hop equated with the hop for networks in that communication energy levels of every sensor is set. The extra period is comparative to the dissimilarity among the highest as well as least quantity of energy for a sensor to communicate a message through correspondingly [Lin and Chen, 2017].

A delay-aware routing with data fusion is introduced structure prepares sensor nodes into clusters of dissimilar sizes thus every cluster can transmit with the fusion center. An optimization procedure is introduced to optimize intra-cluster transaction distance. This approach can decrease delays in data collection procedures also stay the total energy utilization at lower levels offered which data are only incompletely fusible [Cheng et al, 2013]. A cluster-based disseminated data collection scheduling technique, disseminated multi-energy as well as multichannel which can diminishes the data collection delay. In this approach, to preserve the energy less communication energy is applied for communications within a cluster, also great energy is applied for communication between clusters [Ren et al, 2017]. Distributed delay efficient data collection scheduling approach is employed to resolve the aggregation-scheduling difficulty in duty-cycled network [Kang et al, 2017]. Distributed and efficient data aggregation scheduling that diminishes the delay in the combined data via multichannel connections. Initially, this scheduling to schedule sensors to evade intervention as well as reduced the delay of data aggregation. The Markov estimation procedure based adaptative $\beta$ is additional flexible as well as well-organized than the solitary $\beta$ approximation [Goa et al, 2019]. Attribute-aware Data Aggregation (ADA) approach contains of a packet-driven timing method also a particular dynamic routing approach. Encouraged through the idea of possible in physics as well as secretion in ant colony, a potentiality based active routing is detailed to sustain an ADA scheme [Ren et al, 2012].

\section{RELIABLE DATA AGGREGATOR BASED EFFICIENT DATA COLLECTION FOR IMPROVING ENERGY EFFICIENCY}

This approach clusters, the sensor nodes established on the connection as well as data density also it is applied at the $\mathrm{CH}$ to efficiently collective the data. The proficient data collection with node clustering as well as excessive task is utilized to envisage high-dimensional variable data in little dimension in addition eliminate the redundancy. The aim of this approach is to diminish the expenditure of transaction through resourcefully collecting the data as well as transmitting precise data to the BS. The introduced data collection approach 
applies active message list to communicate only precise also compressed data to the BS. This works task in three parts such as cluster formation, $\mathrm{CH}$ selection, Trustable data aggregator Selection, and filtering process. In the initial part, the sensor nodes are forming the clusters by sensor node density [Kumar and Dutta 2016]. The sensor node of the greatest degree as well as adequate energy declares itself as the $\mathrm{CH}$. Afterward the structure of the clusters, every $\mathrm{CH}$ distinguishes several routes to the BS in the spine as well as next chooses the better route for data communication. In the following part, the sensor information is filtered applying active message list in every cluster member node earlier than communicated to the $\mathrm{CH}$. In the final part, the observing information is filtered through convincing the great-dimensional information to low dimensional ones. This approach efficiently diminishes the number of communicated information as well as energy utilization of the nodes, when raising the precision of information.

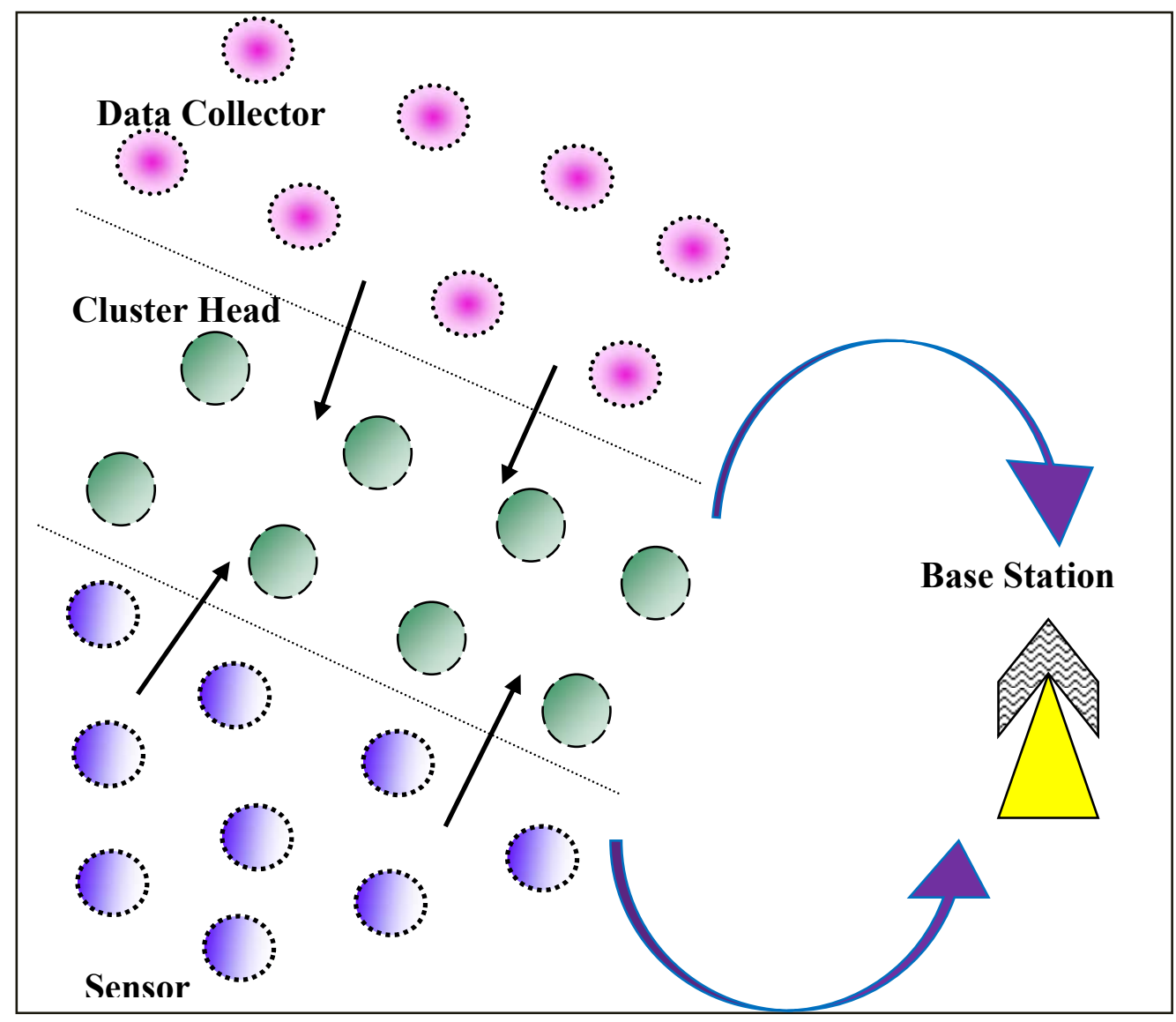

Fig. 1. Architecture of REDC.

In WSN, every sensor node collects the environment information arbitrarily and this similar information will be transmitted via several sensor nodes thus much amount of reduplicated information is presented. Generally, the sensor node lifetime and memory is restricted. Hence, this duplicated information is decreasing the sensor lifetime and node burdens. But this filtering process is utilized to avoid this problem.

A BIM is used for selecting reliable data collector in the WSN. The BTM method like ACO technique achieves an efficient path leading to the most reputed node in a WSN. In this method, each senor consist of pheromone traces. It represents the hormones that decide the possibility for an ant to choose a collector.

In the data collection procedure every low level node transmits their information to its above level, that above level node turn into an collector which collects an entire information upcoming from its subordinate level node and next transmit to its upper level sensor. Here, the node bandwidth, energy, as well as time attributes are used to select the collector by BIM method.

The major benefit of this method is smallest energy utilization because of the procedure of information collection. In addition, this approach checks the collector node reliability by polynomials key verification. The bivariate t-degree polynomials key is computed for every connection present among the sensor $l$, and $m$ is given below. 
$F(c, d)=\sum_{l, m=0}^{r} b_{l m} c^{l} d^{m}$

here, $c, d$ represents the collector key attributes and the coefficients $c_{i j}(0 \leq 1, m \leq r)$ are arbitrarily select from a limited Galois field $\mathrm{GF}(\mathrm{P})$ and $\mathrm{P}$ denotes the prime number.

$$
F(c, \mathrm{~d})=F(d, \mathrm{c}), b_{l m}=C_{m l}
$$

The similar key is applied for transmitting the data from 1 to $\mathrm{m}$ as well as $\mathrm{m}$ to 1 .

Fig.2 explains a polynomial key verification procedure. If the collector sensor confirms the key verification, after the collector sensor transmit the next level till the BS reach the information. Otherwise, the collector node send the notification message to whole WSN network.

In the data collection procedure, the most significant issue is precision filtering and it is the distinctive function can be experimental. It is two feature of this such as precision rate another one is skip rate. The filtering precision rate computation is given below.

$$
\text { Precision Rate }=\frac{\mathrm{RD}_{\text {info }}}{\text { Total } \mathrm{F}_{\text {info }}}
$$

Filtering precision rate is depicts that the ratio of amount of reduplicated information and the amount of total filtering information in the network. The skipping rate is depicts the ratio of amount of information that could be filtered out except be communicated and the amount of total reduplicated information.

$$
\text { Skip Rate }=\frac{F_{\text {info }}}{\text { Total } \mathrm{RD}_{\text {info }}}
$$

Filtering procedure contains three steps: initially filtering nodes are positioned in CHs also primary active list is established; Next, the environment information is filtered as well as communicated through $\mathrm{CH}$ nodes; Finally, the distance of list should be active reorganized along with the list's satisfied simplified state.

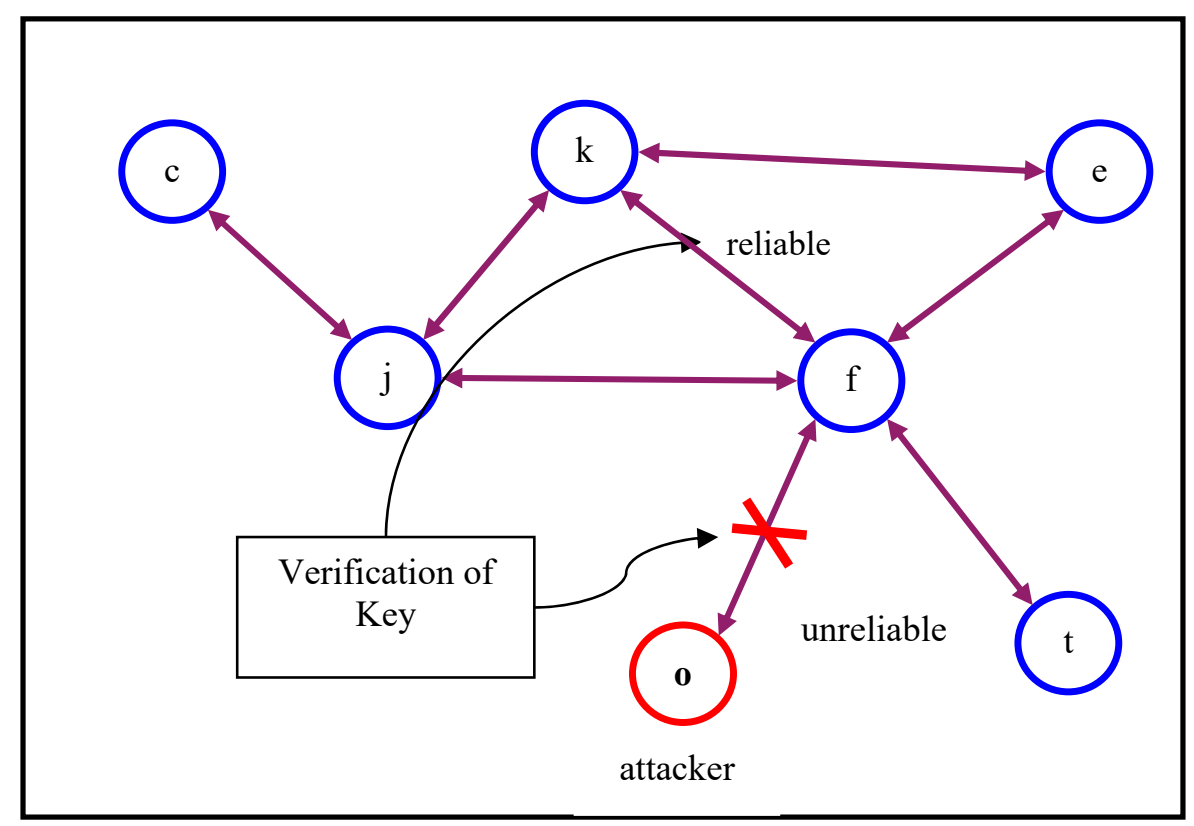

Fig. 2. Data Collector Verification Procedure.

Step1: filtering node position also generating preliminary list: Generally, $\mathrm{CHs}$ are arbitrarily disseminated, the dissimilar nodes' communicated load is a large amount and the $\mathrm{CH}$ nodes closer to destination node has to take on several jobs to communicate information from external region as well communicating its member nodes information. To adjust this happening, this approach presets placing on each filtering node whose value are determined through the hops amount from this node to destination. The procedure of this function is as:

To begin with, the highest hops of every node $H_{\text {Level }}$ will be revealed through placing entire clusters; next the message list's distance of every filtering node is calculated given below. 
List $_{\mathrm{j}}$. dis tan ce $=\mathrm{H}_{\text {level }}-\mathrm{CH}_{\mathrm{j} \text {.level }}+1$

List $_{\mathrm{j}}$. flag $=$ dis tan ce

Here, $\mathrm{CHj}$. Level is the hops amount of $\mathrm{CH}$ with Identity j, flag represents the variable denotation the frequency of information in present thing of List $_{j}$, and lifespan is a variable denotation the period intermission from present time to end time that obtained similar information. And these two variables are utilized to manage the dynamical alteration of present list. While $\mathrm{CH}$ node has been resolved, the list should be also communicated to recent $\mathrm{CH}$.

Step2: Filtering and transmitting message: Network will go to communicating stage, with in this stage; several repeated duplicated messages will be created, thus need proficient filtering function.

Here, $\mathrm{CH}$ will rapidly achieve the filtering function while it obtains a message. The filtering node $\mathrm{j}$ will look around its list, and then obtained information is being compared with each item of List $t_{\mathrm{j}}$. If there is not any items' satisfied equalling to the present information, this information will be communicated at once; next this information will be upended to the list.

Active adjusting the list's length: Here, the function is to energetically revise the length of information list. To conclude this task, twice thresholds are distinct: one is to referee whether dilutes the distance of list; the other is to referee whether extends the distance of list.

\section{Simulation Analysis}

The REDC through itself can be utilized as a proficient routing metric because it efficiently catches not only the delay of the queue; however, the retransmission delays. We employ NS2.35 simulation results for a WSN. Here, the 50 sensor nodes are arbitrarily distributed in a $600 \times 500 \mathrm{~m}^{2}$ topology area with required modification to preserve the property. The node communication range is $200 \mathrm{~m}$, the size of the packet is 512 bytes, and we utilized constant-bit-rate flow.

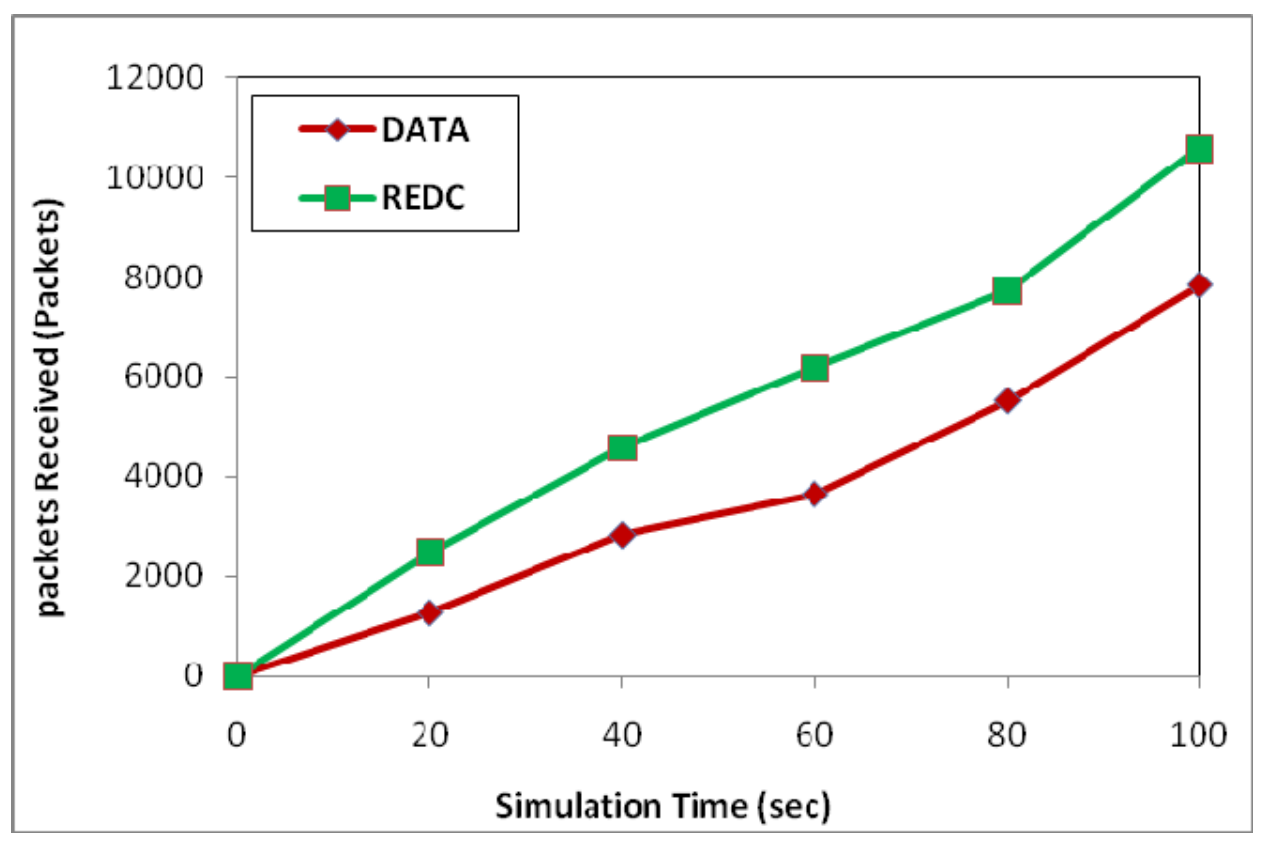

Fig. 3. Packet Received of DATA and REDC Scheme.

Packet received rate is represented as the part of the total of effective packet transmitted to the amount of packets received. Fig. 3 illustrates the packet received rate of REDC and DATA scheme. The REDC scheme equates to the DATA, the REDC offer better packet received rate in the network. Since, REDC scheme select the collector node by ACO optimal method. Therefore, increase the routing efficiency.

Fig. 4 shows the average delay rate explicitly around recognized with the time period essential to distribute the whole data. This figure proves the REDC have less delay time than the DATA scheme. Because of REEC scheme verifies the collector node through the bivariate polynomial key verification. So, REDC approach transmit the data via reliable collector node. 


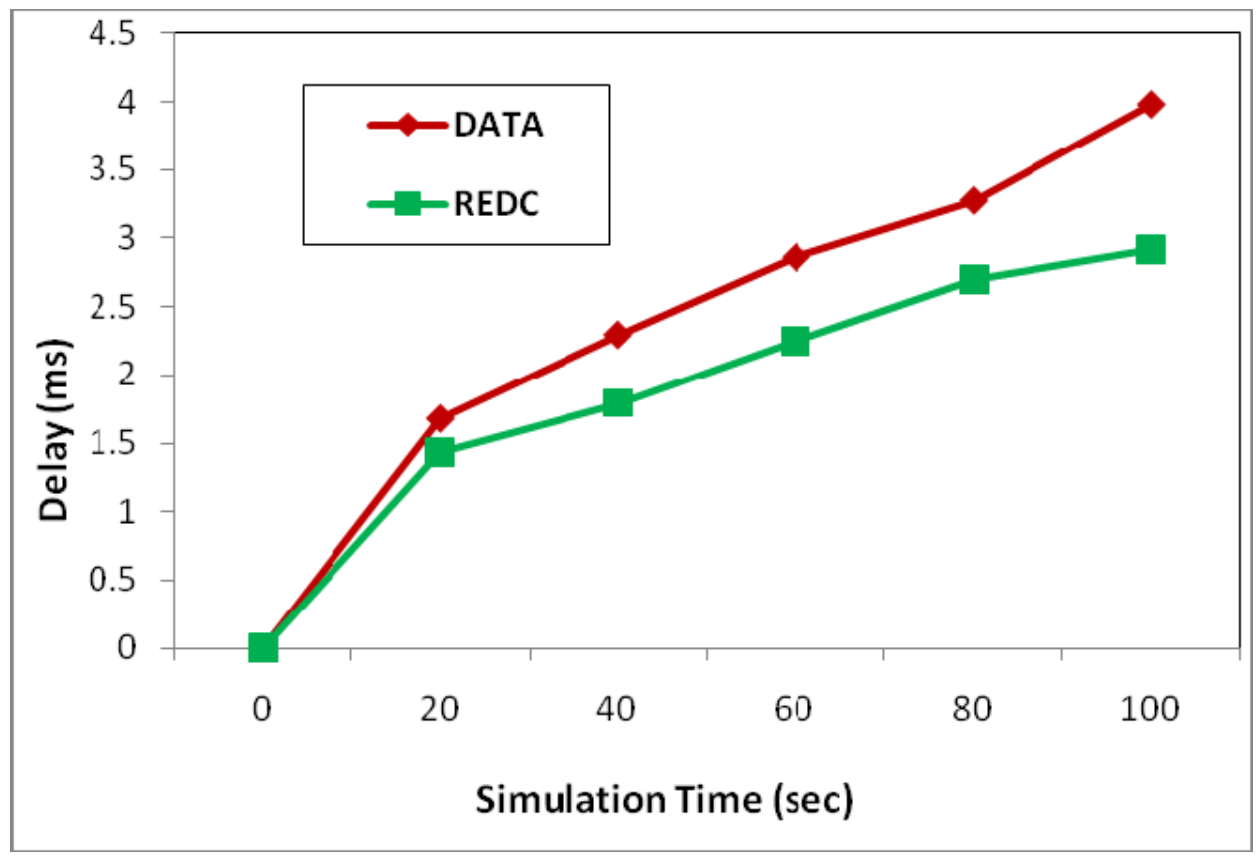

Fig. 4. Delay of DATA and REDC Scheme.

Fig. 5 demonstrates the Packet loss of REDC and DATA scheme. The REDC scheme diminishes the loss of data packet since it chooses to $\mathrm{CH}$ by distance, energy and delay in the network. But, DATA has more packet losses. Thus, DATA increases the packet losses in the network.

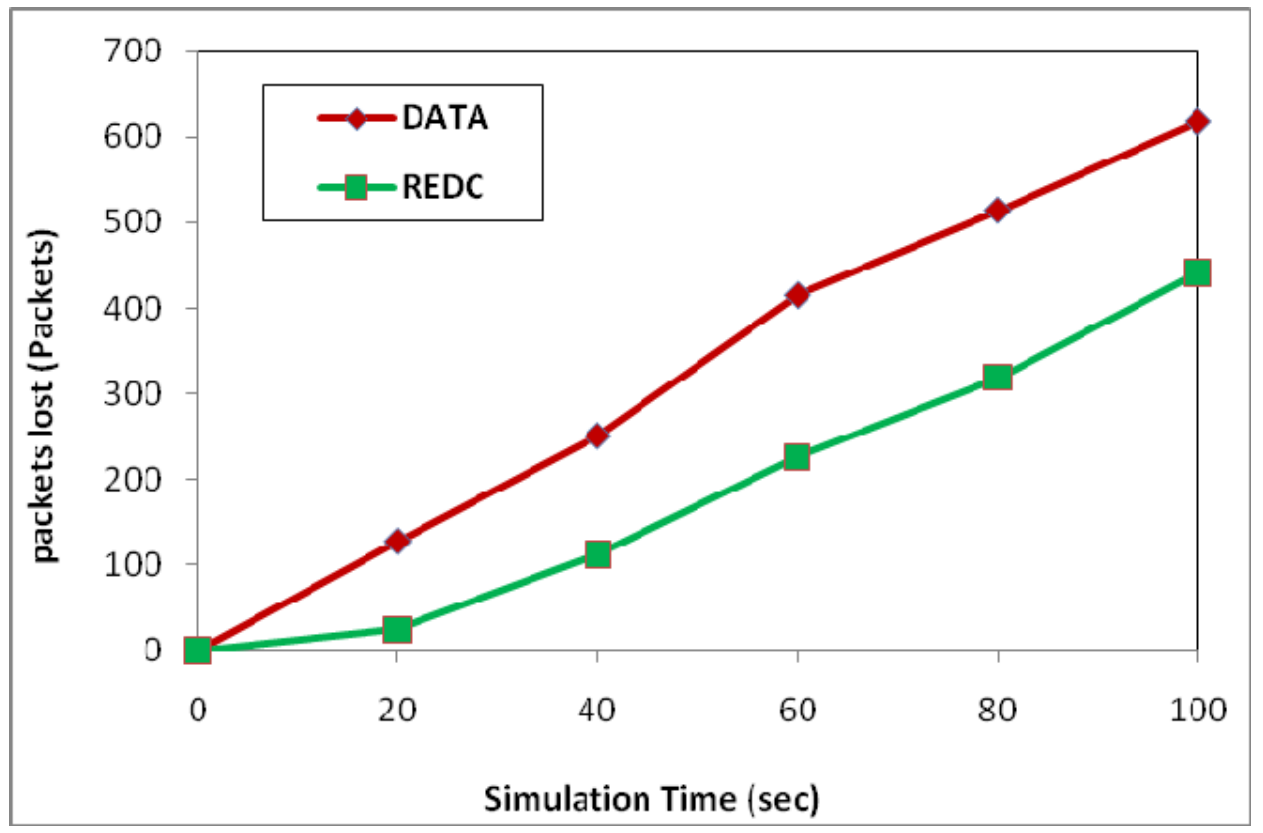

Fig. 5. Packet Loss of DATA and REDC Scheme.

Remaining Energy represents the amount of energy remaining in a network. Fig. 6 illustrates the Remaining energy of REDC and DATA scheme. The REDC scheme equates to the DATA, the REDC offer better remaining energy since the $\mathrm{CH}$ forward the data through reliable nodes. As a result enhances the remaining energy in the network. 


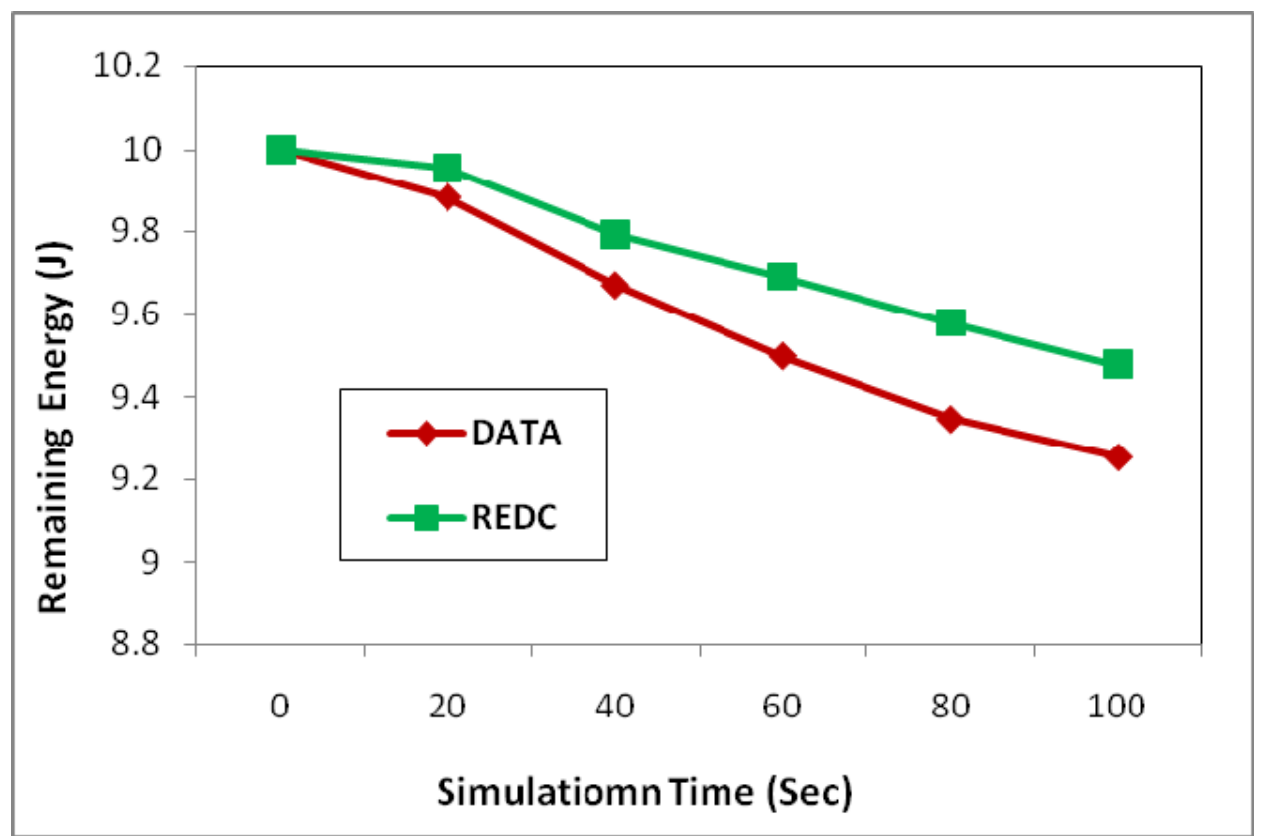

Fig. 6. Remaining Energy of DATA and REDC Scheme.

Fig. 7 explains an data collection accuracy of DATA and REDC approach. In REDC, unreliable nodes are detected by bivariate polynomial key verification method. ACO method also increased the routing efficiency. As a result, REDC approach increases the accuracy compared to the DATA approach.

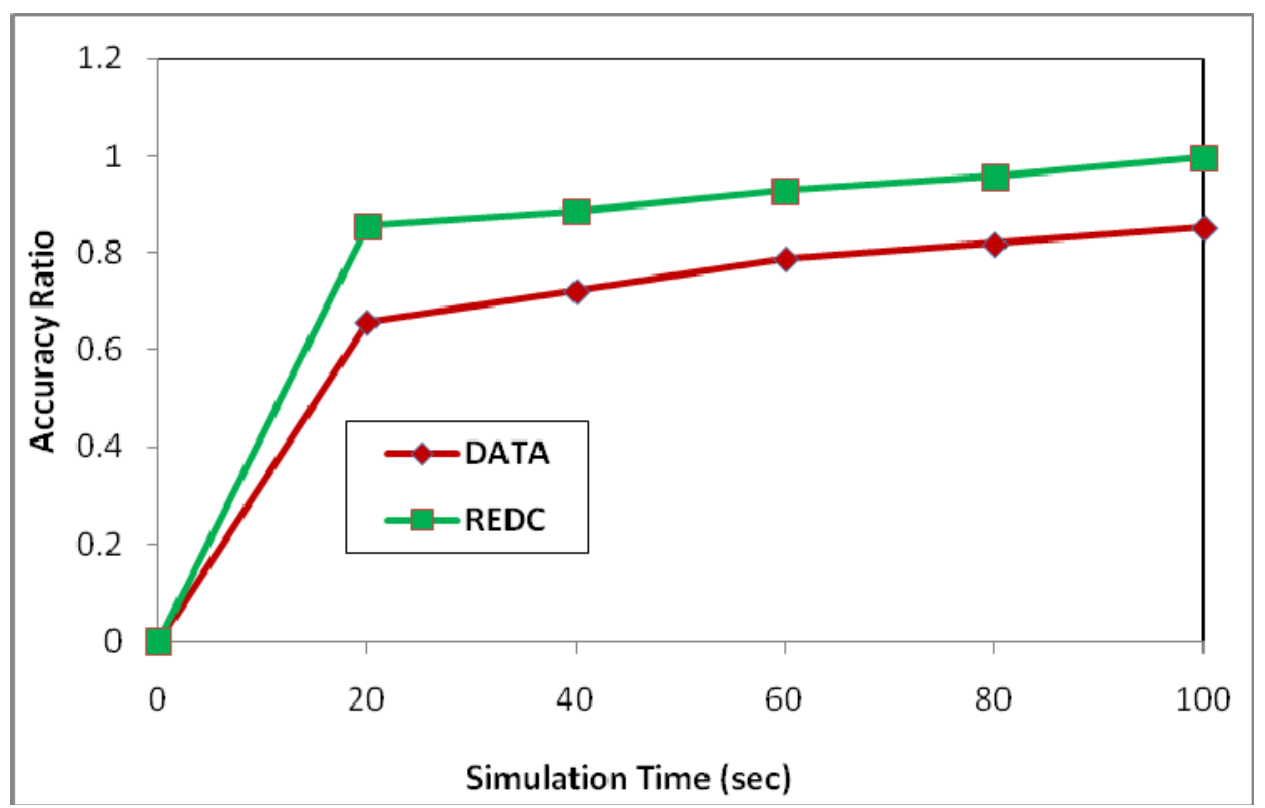

Fig. 7. Accuracy Ratio of DATA and REDC Scheme.

\section{Conclusion}

In this paper, the communication expenditure. In this paper, an Reliable Data Collector based Efficient Data Collection for Improving Energy Efficiency in WSN. The ACO method is used for selecting reliable data collector and it can achieved an efficient path. Bivariate polynomials key verification is used for verified the reliable collector. Active list will be generated in filtering node to accumulate history messages yet communicated through this node. The entire messages will be estimated whether repeated duplicated or not along with the messages in list. Thus, traffic of network can be considerably diminished that in revolve expands the lifespan. The simulation results demonstrate which the REDC scheme improved an accuracy of data collection and it can raised a remaining energy. In addition, REDC scheme minimized both the delay and the packet loss rate. 


\section{Funding Information:}

No funding agency.

\section{References}

[1] Haghighi, M. S.; Xiang, Y.; Varadharajan, V.; Quinn, B. (2014): A stochastic time-domain model for burst data aggregation in IEEE 802.15. 4 wireless sensor networks. IEEE Transactions on Computers, 64(3), pp. 627-639.

[2] Roy, N. R.; Chandra, P. (2019): EEDAC-WSN: Energy Efficient Data Aggregation in Clustered WSN. In 2019 International Conference on Automation, Computational and Technology Management, pp. 586-592. IEEE.

[3] Waghmare, K. A.; Chatur, P. N.; Mathurkar, S. S. (2016): Efficient data aggregation methodology for Wireless Sensor Network. IEEE International Conference on Wireless Communications, Signal Processing and Networking, pp. 137-139.

[4] Miranda, K.; Ramos, V. (2016); Improving data aggregation in Wireless Sensor Networks with time series estimation. IEEE Latin America Transactions, 14(5), pp.2425-2432.

[5] Sasirekha, S.; Swamynathan, S. (2017): Cluster-chain mobile agent routing algorithm for efficient data aggregation in wireless sensor network. Journal of Communications and Networks, 19(4), pp. 392-401.

[6] Campobello, G.; Serrano, S.; Galluccio, L.; Palazzo, S. (2013): Applying the Chinese remainder theorem to data aggregation in wireless sensor networks. IEEE communications letters, 17(5), pp. 1000-1003.

[7] Lin, H. C.; Chen, W. Y. (2017): An approximation algorithm for the maximum-lifetime data aggregation tree problem in wireless sensor networks. IEEE Transactions on Wireless Communications, 16(6), pp.3787-3798.

[8] Cheng, C. T.; Leung, H.; Maupin, P. (2013): A delay-aware network structure for wireless sensor networks with in-network data fusion. IEEE Sensors Journal, 13(5), pp.1622-1631.

[9] Ren, M.; Li, J.; Guo, L.; Li, X.; Fan, W. (2017): Distributed data aggregation scheduling in multi-channel and multi-power wireless sensor networks. IEEE Access, 5, pp.27887-27896.

[10] Wan, S.; Zhang, Y.; Chen, J. (2016): On the construction of data aggregation tree with maximizing lifetime in large-scale wireless sensor networks. IEEE Sensors Journal, 16(20), pp.7433-7440.

[11] Kang, B.; Nguyen, P. K. H.; Zalyubovskiy, V.; Choo, H. (2017): A distributed delay-efficient data aggregation scheduling for dutycycled WSNs. IEEE Sensors Journal, 17(11), pp.3422-3437.

[12] Gao, Y.; Li, X.; Li, J.; Gao, Y. (2019): Distributed and Efficient Minimum-Latency Data Aggregation Scheduling for Multichannel Wireless Sensor Networks. IEEE Internet of Things Journal, 6(5), pp.8482-8495.

[13] Ren, F.; Zhang, J.; Wu, Y.; He, T.; Chen, C.; Lin, C. (2012). Attribute-aware data aggregation using potential-based dynamic routing in wireless sensor networks. IEEE transactions on parallel and distributed systems, 24(5), pp. 881-892.

[14] Nakas, C.; Kandris, D.; Visvardis, G. (2020): Energy efficient routing in wireless sensor networks: a comprehensive survey. Algorithms, 13(3), pp. 72, 2020.

[15] Bista, R.; Chang, J.W. (2010): Privacy-preserving data aggregation protocols for wireless sensor networks: a survey. Sensors, 10 (5), pp.4577-4601, 2010.

[16] Kumar, M.; Dutta, K. (2016). LDAT: LFTM based data aggregation and transmission protocol for wireless sensor networks. Journal of Trust Management, 3(1), pp. 1-20.

[17] Raychaudhuri, A.; De. D. (2020): Bio-inspired algorithm for multi-objective optimization in wireless sensor network. In Nature Inspired Computing for Wireless Sensor Networks, pp. 279-301, Springer, Singapore.

[18] Shayokh, M.; Shin, S.Y; (2017): Bio inspired distributed WSN localization based on chicken swarm optimization. Wireless Personal Communications, 97(4), pp. 5691-5706.

\section{Authors Profile}

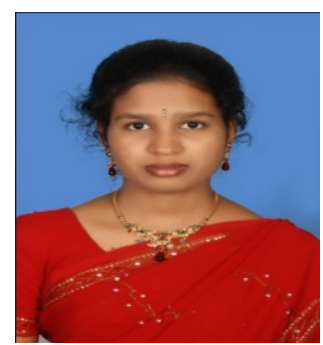

G. Vishnupriya, received her B.E. degree in computer Science and Engineering from Madha engineering College, affiliated to Anna University in the year 2011. She completed her M.E degree in Computer science and Engineering from Sathyabama University in the year 2013. She is currently pursuing her Ph.D. in Sathyabama Institute of Science and Technology under the Faculty of Computer Science and Engineering. She is working as Assistant Professor in the Department of Computer Science and Engineering, Jeppiaar Maamallan Engineering College, Chennai. Her research interests include Computer Networks, Wireless Communications, Security issues of wireless networks and routing protocols in ad hoc networks.

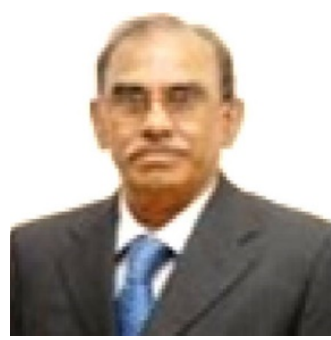

R. Ramachandran is a graduate in Telecommunication Engineering, Post graduate in Communication Systems (Anna University, Chennai) and obtained Ph.D. (Anna University, Chennai) in "Optical Neural Networks". He has served initially for 20 years in telecommunication field in a Government of India organization. After this he joined as a faculty in the department of Electronics and communication Engineering, Sri Venkateswara College of Engineering, Sriperumbudur and served for 25 years. He is also former Director (Research), Sri Venkateswara college of Engineering, Sriperumpudur, Chennai, India. He is also Research supervisor, Sathyabama Institute of Science and Technology, Chennai, India. 